\title{
Leprosy and Primary Health Care
}

\author{
W F ROSS \\ American Leprosy Missions, Inc, 1262 Broad Street, Bloomfield, \\ New Jersey 07003, USA
}

Received for publication 9th March 1981

\section{How much patient care can we expect?}

If all goes well with the development of 'Health Care for All by the year 2000', and current estimates of the prevalance of leprosy are approximately correct, and no dramatic changes take place in leprosy control technology, then we can expect that the number of leprosy patients under treatment will triple to about 12 million in the next 19 years. Clearly, most of the work for treating these cases will have to be done by Primary Health Care (PHC) workers. This need not be the disaster some fear - it could indeed be the greatest thing ever to happen for the cause of leprosy control - provided the leprosy health professionals grasp the opportunity being presented. The challenge to us is to determine exactly what role PHC workers can play in leprosy patient care, and to set out to train them - or more likely train others to train them - to fulfill that role. This paper defines a role for PHC workers in leprosy patient care, and outlines the essential components of a training programme to equip them to fulfill that role. It is based on the assumptions that PHC workers will be supported by an effective system of supervision, and will have the possibility of referring cases beyond their competence to a formal health service unit. It also assumes that while PHC worker trainees may not be fully literate, even in a vernacular, they will at least be able to recognize colour photographs as an acceptable abstraction of reality. This paper is not definitive. It is introductory. It is a hypothesis intended to stimulate experimentation and the development of a valid theory and practice in PHC worker training in leprosy.

\section{What can we expect?}

If $\mathrm{PHC}$ workers are to continue to contribute anything to leprosy patient care and leprosy patient control it is reasonable to expect them to have the following competencies: 
1 Willingness to accept leprosy patients as people.

2 Ability to recognize leprosy cases.

3 Ability to recognize complications of leprosy in known cases.

4 Ability to refer cases with leprosy or its complications promptly for diagnosis and management of leprosy and its complications.

5 Ability to dispense prescribed medicines regularly to patients.

6 Ability to train patients in self care.

\section{What training will be required to enable PHC workers to acquire these competencies?}

\section{COMPETENCY}

Willingness to accept patients as people is a general social skill through which the PHC worker will accept all people, irrespective of the disease from which they suffer, as human beings with equal rights on the basis of their common humanity. However, because of the peculiar position of leprosy patients in many cultures, special training may be required in this area. The underlying attitude to patients upon which this competency depends can only be taught effectively by those who are themselves convinced of its importance and demonstrate their own adherence to its truth. The measures needed to produce such teachers will vary from culture to culture, and even from individual to individual. A detailed knowledge of specific, and even local situations, will be necessary, and perhaps the most significant responsibility of leprosy health professionals, with respect to the PHC concept, is to change the attitude of a whole profession to leprosy, and insure that patients with the disease are accepted as people throughout the medical service system. There will be no better place to begin this task than the medical schools and schools for training teachers for paramedical workers. For it seems a reasonable assumption that it is upon doctors and paramedical teachers that the main burden of training PHC workers will fall.

\section{COMPETENCIES 2 AND 3}

The recognition of leprosy cases and of the complications of leprosy are specific to this disease. These competencies can be acquired even by illiterate people by means of the study of appropriate pictures in colour, reasonably explained in the local vernacular, for as has been shown in the case of smallpox, the use of posters can enable even illiterate villagers to recognize cases. There will necessarily be some degree of over recognition and under recognition. The former should not be a serious problem if reasonable attitudes are taken towards leprosy patients, and the latter will certainly be very much less than it would be if no training were given to villagers in the recognition of the disease. 
COMPETENCIES 4 AND 5

Prompt referral of cases for diagnosis and for the management of complications, and the regular dispensing of prescribed medicines are general competencies needed by the PHC worker for the care of patients with other diseases. In respect to leprosy, he will simply be required to be able to read instructions concerning the quantity and frequency of distribution of medicine and must be informed of the place, date, and time of clinics where leprosy cases may be seen.

\section{COMPETENCY 6}

Patient education and self care will be the most difficult competency for PHC workers to acquire, but it may also be the most important. It may be argued that patient education in self care should be the responsibility of the professional health system staff, but since the patients will only be seen for diagnosis, and if complications arise, and for periodic review by the professional staff, it would seem more appropriate for the PHC worker to be responsible for patient education if at all possible. Patient self health care has 7 components.

1. Understanding the importance of regular treatment.

2. Early recognition of reactive phenomena in skin and nerves and eye problems.

3. Early recognition of open and closed wounds.

4. Recognition of the presence of anaesthesia, anhidrosis, and weakness in face, hands, and feet.

5. Self-reporting, promptly, of reactive phenomena, eye problems, and severe open wounds.

6. Home management of closed and of minor open wounds.

7. Home management of anaesthetic, anhidrotic, weak and/or paralysed limbs.

Appropriate teaching processes for PHC workers to use in their work with patients are as follows:

1. The importance of regular treatment may be taught by means of a series of colour pictures of patients illustrating the results of regular and irregular treatment. The patients should also be informed that their compliance with instructions will be checked by the PHC worker at intervals.

$2 \& 3$. Early recognition of reactive phenomena, eye problems, and of open and closed wounds can be taught by means of suitable colour pictures for showing to patients.

4. Recognition of the presence of anhidrosis, anaesthesia and weakness may best be taught to the PHC workers by demonstration of patients 
with these problems. Similarly, it can be taught to the patients themselves. If there are no patients available for teaching, then photographs may be used as a substitute.

5. The step between recognition of problems and early self-reporting will only be taken by patients if the PHC worker has the confidence of the patients. The PHC workers must be taught to earn this confidence through treating their patients with courtesy, through competence in their work, and through their reliability and accessibility to their patients.

$6 \& 7$. Home management of closed and minor open wounds, and of anaesthetic, anhidrotic, and weak or paralysed limbs can best be taught to paramedicals by actual demonstration of cases and practice of home management skills under supervision. In the absence of cases, photographs can be used to illustrate the process and simulated practice undertaken by the health care workers, using their own limbs as models.

\section{The role of leprosy professionals}

We should be preparing now to provide the type of teaching material outlined above, suitably adapted to meet specific local needs. In particular, it is most important for any pictures produced to be in keeping with local culture and not to be obscured or complicated with extraneous information. The urgency and importance of this task, in relation to the training of PHC workers who are both safe and effective in leprosy control, cannot be over-rated. 